\title{
A strong ice cloud event as seen by a microwave satellite sensor: Simulations and observations
}

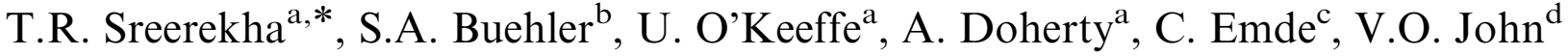 \\ ${ }^{\mathrm{a}} U K$ Met Office, Exeter, UK \\ ${ }^{\mathrm{b}}$ Department of Space Science, Lulea Technical University, Kiruna, Sweden \\ ${ }^{\mathrm{c}}$ German Aerospace Center, DLR, Oberpfaffenhofen, Germany \\ ${ }^{\mathrm{d}}$ RSMAS, University of Miami, Miami, USA
}

Received 28 August 2007; received in revised form 30 November 2007; accepted 23 December 2007

\begin{abstract}
In this article, brightness temperatures observed by channels of the Advanced Microwave Sounding Unit-B (AMSU-B) instrument are compared to those simulated by a radiative transfer model, which can take into account the multiple scattering due to ice particles by using a discrete ordinate iterative solution method. The input fields, namely, the pressure, temperature, humidity, and cloud water content are taken from the short range forecast from the Met Office mesoscale model (UKMES). The comparison was made for a case study on the 25 January 2002 when a frontal system associated with significant cloud was present over the UK. It is demonstrated that liquid clouds have maximum impact on channel 16 of AMSU whereas ice clouds have maximum impact on channel 20. The main uncertainty for simulating microwave radiances is the assumptions about microphysical properties, such as size distribution, shape and orientation of the cloud particles, which are not known in the mesoscale model. The article examines the impact of these parameters on the cloud signal. The polarisation signal due to oriented ice particles at these frequencies is also discussed.

(C) 2008 Elsevier Ltd. All rights reserved.
\end{abstract}

Keywords: Clouds; Scattering; Radiative transfer; Polarisation

\section{Introduction}

Microwave remote sensing from space is a key technique to study the weather and climate of the Earth. The Advanced Microwave Sounding Unit-A (AMSU-A) and AMSU-B on-board the NOAA satellites and the Special Sensor Microwave Imager (SSM-I) and SSM-T2 on-board the Defense Meteorological Satellite Program (DMSP) satellites have been used, mainly for the retrieval of temperature and water vapor $[1,2]$. The AMSU data are also used for operational weather forecasts by assimilating radiances into the numerical weather prediction (NWP) models [3].

\footnotetext{
*Corresponding author.

E-mail address: tr.sreerekha@metoffice.gov.uk (T.R. Sreerekha).

URL: http://www.metoffice.gov.uk/ (T.R. Sreerekha).
} 
Even though clouds have a smaller impact on microwave radiation compared to visible or infrared radiation (Greenwald and Christopher [4], based on their results for channel $18(183.31 \pm 1 \mathrm{GHz})$ of AMSU estimated the effect of non-precipitating clouds on this channel to be measurable, but rather weak, about $1.4 \mathrm{~K}$ in average), several modelling and observational studies at microwave wavelengths suggested that the presence of cirrus clouds associated with strong weather activity can severely disturb the measurements in the microwave region [4-8]. The main interaction of ice particles in cirrus clouds with microwave radiation (considered in this study, 89-200 GHz) is by scattering, which decreases the brightness temperature (BT) compared to the clear sky case. A detailed simulation study which examined the impact of clouds on microwave frequencies for down-looking instruments is presented in Sreerekha [9]. There, the effect of non-spherical, oriented ice particles and polarisation signal from such particles on AMSU-B channels using a newly developed radiative transfer (RT) model, the atmospheric radiative transfer simulator (ARTS), was shown. These studies pointed out that the cloud signal depends on the cloud microphysical properties and relative water vapor absorption at the AMSU-B frequencies.

This study presents a comparison of the BTs simulated by the RT model ARTS and those observed by AMSU-B. ARTS is a polarised three-dimensional (3D) vector RT model that can simulate multiple scattering due to spherical or non-spherical oriented ice particles in cirrus clouds. The ARTS model has been compared against other scattering models [10-13]. In all those comparisons ARTS was the reference model, because all of the other RT models use different kinds of approximations, e.g. plane-parallel geometry, single scattering approximation or neglect of polarisation. All models agreed well in the expected regimes where the approximations are valid.

There are only a limited number of in situ observations of cloud microphysics that are coincident with the AMSU-B observations, therefore it is difficult to get model input data for a radiative transfer simulation of the AMSU observation. In this study we use atmospheric fields, including all relevant cloud fields, from a NWP model. The Met Office mesoscale model (UKMES) was chosen because it has high spatial resolution (12 km) and short range forecasts (between 3 and $9 \mathrm{~h}$ ) were taken to give the cloud scheme time to produce realistic cloud and precipitation fields from the original analysis and to achieve as close a match in time as possible to the observations. The result of this comparison will on the one hand side provide information about how much clouds affect the different AMSU channels and on the other hand side they can be used for validation of the NWP model, the results show how closely the short range forecasts the AMSU observation.

A similar study was done by Burns et al. [7], where the results of the RTM RT model were compared to SSM-T2 measurements using input from the Cooperative Institute for Meteorological Satellite Studies (CIMSS) forecast model [14] for a convective system over the Pacific Ocean on 9 February 1995. In that study, the BTs simulated in the presence of ice clouds did not show the BT depression found in the observation. The reason was attributed to the coarse resolution of the forecast model of about $80 \mathrm{~km}$. The authors came to this conclusion after using the high resolution input fields provided by the University of Wisconsin Regional Atmospheric Modeling System (UW-RAMS) cloud resolving model (CRM) [15] for Hurricane Gilbert on September 1988, which produced BT depressions of the magnitude observed for the previous case study. However, a direct comparison with the CRM inputs for the original case study was not done.

Meirold-Mautner et al. [16] also describe a comparison between observed and simulated radiances for AMSU-B frequencies, but for quite different meteorological conditions. Furthermore, they based their inputs on a different circulation model, and used a different RT model. Despite these differences, we show in Section 6.2 that our results are broadly comparable to their results.

The article is organised as follows: Section 2 gives a short description on the ARTS RT model, Section 3 describes the UKMES, and Section 4 introduces the AMSU-B instrument. Section 5 explains the methodology of the comparison, Section 6 discusses the results of the comparison between ARTS simulation and AMSU-B observation in detail, and Section 7 contains the summary and conclusions.

\section{The atmospheric RT simulator}

The first version of the ARTS is a clear sky RT model, used for the retrieval of temperature and trace gases in the microwave range. ARTS is a very flexible and modular tool that can be used for simulating up-, limb-, and down-looking instruments. ARTS operates in 3D geometry which makes it suitable for limb and highly 
off-nadir measurements. Because cirrus clouds interact with microwave radiation mainly by scattering, the model was extended to include multiple scattering. In order to take into account the polarisation due to scattering by non-spherical oriented ice particles in cirrus clouds, the vector radiative transfer equation (VRTE) is solved in ARTS. Two methods to solve the VRTE are implemented in ARTS: a discrete ordinate iterative (DOIT) method [17] and a Monte Carlo (MC) method [18]. In this study we use the DOIT method since it is efficient in 1D geometry. We take the cloud fields of the NWP model as independent columns for the radiative transfer simulations, this methodology is commonly known as independent pixel approximation. A real 3D simulation of the observation is not yet feasible in reasonable CPU time.

Validation of RT models including scattering is difficult, because the model input data (i.e., cloud structures, particle sizes, particle shapes, etc.) are not well known. Therefore one cannot directly compare measured BTs to the simulations. One possibility to validate RT models is by comparison to other models. The two methods implemented in ARTS (DOIT method and MC method) are two completely different approaches to solve the VRTE. They have been compared and agreed well within the expected numerical errors which gives very high confidence in the two algorithms [11].

ARTS-DOIT has been used to validate several other RT models that use various kinds of approximations: a comparison with the RT model developed at RAL (Rutherford Appelton Laboratory), which is a pseudospherical model that approximates the scattering phase function by a Henyey-Greenstein function, showed excellent agreement, less than $1 \mathrm{~K}$ difference in simulated BTs for most cloud cases in limb-looking geometry [10]. Another comparison between ARTS-DOIT and the single scattering model Karlsruhe Optimized and Precise Radiative Transfer Algorithm (KOPRA), in the infrared region, showed that the two models agreed well in the single scattering regime [12]. ARTS has also been used to validate the pseudo-spherical model SARTE [13].

The single scattering properties of cloud particles are calculated using the T-matrix code for randomly oriented particles [19]. The real and imaginary part of the refractive index, which are inputs to the T-matrix code are calculated using Warren [20] for ice particles and Mishchenko and Travis [19] for liquid droplets.

\section{The mesoscale model-UKMES}

The UK Met Office runs an operational mesoscale model, which assimilates data from satellite, radiosonde, aircraft and surface stations. Compared to the global model of the UK Met Office, the mesoscale model provides additional mesoscale details of clouds, precipitation, wind, and temperature over the UK. The global model is used to provide the lateral boundary conditions. The UKMES model uses 3D-var to assimilate conventional observations as is described for the Met Office global model [21]. AMSU data are also assimilated by the UKMES but has been excluded in the studies in this paper. The model also assimilates radar data and infrared satellite data using an analysis correction scheme. The model has a horizontal grid spacing of $0.11^{\circ}$ corresponding to approximately $12 \mathrm{~km}$ and there are 38 vertical levels.

Of the outputs of the mesoscale model, the ones that are of interest in this study are pressure, temperature, relative humidity, cloud ice content, and cloud liquid content. The model output does not contain information on the size distribution of the cloud particles nor their shape or orientation. These must therefore be diagnosed from the model fields.

\section{The AMSU-B instrument}

AMSU-B is a cross-track scanning, total power microwave radiometer with five double side band channels. Two channels are located at 89 and $150 \mathrm{GHz}$, designated as channels 16 and 17, respectively. The other three are located near the $183.31 \mathrm{GHz}$ water vapor line, at $183.31 \pm 1, \pm 3$, and $\pm 7 \mathrm{GHz}$. These are designated as channels 18, 19, and 20, respectively [22]. Fig. 1 shows the atmospheric zenith opacity calculated considering $\mathrm{H}_{2} \mathrm{O}, \mathrm{O}_{2}, \mathrm{~N}_{2}$, and $\mathrm{O}_{3}$ based on the FASCOD mid-latitude summer atmospheric scenario [24]. The vertical light grey bands indicate the pass-band positions of the five AMSU-B channels.

AMSU-B has a field of view of $1.1^{\circ}$, and once every $\frac{8}{3} \mathrm{~s}$ it measures 90 Earth views, four space views and four internal blackbody target views. The Earth viewing angles range from $-48.95^{\circ}$ to $+48.95^{\circ}$ about nadir. The satellite viewing angles for the innermost scan positions are $\pm 0.55^{\circ}$ from nadir, and the viewing angles for 




Fig. 1. Atmospheric zenith optical depth (or opacity) for the FASCOD midlatitude summer scenario for $\mathrm{H}_{2} \mathrm{O}$ (dashed), $\mathrm{O}_{2}$ (dotted), $\mathrm{N}_{2}$ (dash dotted), and total (long-dashed). Shaded regions represent the pass-band positions of channels 16-20 of AMSU. The channel numbers are printed near the pass-bands. Figure adapted from John and Buehler [23].

the outermost scan positions are $\pm 48.95^{\circ}$ from nadir. This corresponds to incidence angles of $\pm 0.62^{\circ}$ and $\pm 58.5^{\circ}$ at the surface, respectively. The footprint size is $20 \times 16 \mathrm{~km}^{2}$ for the innermost scan positions, but increases to $64 \times 27 \mathrm{~km}^{2}$ for the outermost scan positions [22].

In the case of AMSU-B, the vertically and the horizontally polarised radiation cannot be separated. The total radiation as seen by the instrument, $T_{b_{\text {mix }}}$, is given by

$$
T_{b_{\text {mix }}}=T_{b_{\mathrm{v}}} \sin ^{2} \theta_{\mathrm{p}}+T_{b_{\mathrm{h}}} \cos ^{2} \theta_{\mathrm{p}}
$$

where $T_{b_{\mathrm{v}}}$ and $T_{b_{\mathrm{h}}}$ are the vertical and the horizontal polarisation components of the radiation. The polarisation angle $\theta_{\mathrm{p}}$, is the angle between the propagation of the electric field vector and the satellite velocity vector and is given by $\theta_{\mathrm{p}}=90^{\circ}-\theta_{\mathrm{N}}$, where $\theta_{\mathrm{N}}$ is the nadir angle. At nadir, $\theta_{\mathrm{p}}$ is $90^{\circ}$ and AMSU-B is sensitive only to vertical polarisation where the electric field vector is perpendicular to the direction of satellite motion. The ratio of the horizontal to the vertical polarisation increases as the zenith angle $\theta$ moves away from nadir [22].

\section{Set-up and methodology}

For the comparison, a frontal system over UK on 25 January 2002 was chosen as a case-study. The Advanced Very High Resolution Radiometer (AVHRR) infrared image for the same day at 1332 UTC over the region of interest is shown in Fig. 2. This particular day was marked by heavy cloud cover and rainfall over northwest Europe and across the Atlantic as is seen in the figure.

The input fields required for ARTS simulations are taken from the UKMES model, more specifically from its $\mathrm{T}+4$ forecast field from the 0900 UTC run. The mesoscale model output is projected onto the AMSU-B grid. Corresponding to each grid point, profiles of temperature, humidity, ice water content, and liquid water content are given and are used as inputs for the RT calculation. The gaseous species considered are water vapor, oxygen, and nitrogen. The absorption coefficients are calculated according to Rosenkranz [25] for water vapor and oxygen, and Rosenkranz [26] for nitrogen. The column integrated ice water path (IWP) and the liquid water path (LWP) predicted by the mesoscale model are shown in the top right and left panels of Fig. 4, respectively. 


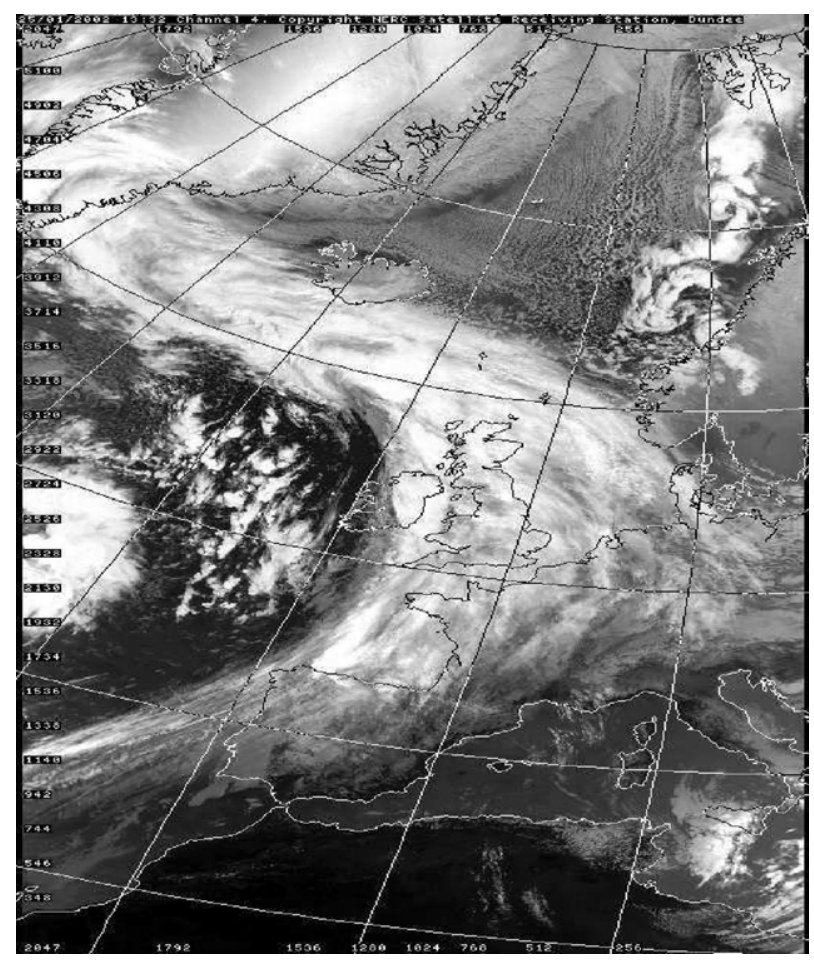

Fig. 2. AVHRR infrared image over the northwest Europe and the Atlantic for 25 January 2002 at 1332 UTC.

All ice hydrometeor types like cloud ice, snow, graupel and hail are treated collectively as a single hydrometeor type following the parameterisation by McFarquhar and Heymsfield [27] where the size distribution is derived as a function of temperature and ice water content. All particles are assumed to be spherical.

The emissivity over ocean is calculated using the surface emissivity model FASTEM [28]. This model calculates the surface emissivity over ocean as a function of frequency, viewing angle, surface temperature and surface wind fields. Over land, a constant emissivity of 0.95 was assumed. Both land and ocean surfaces are assumed to be specularly reflecting. The surface temperature was set to the temperature of the lowest level of each profile. The topographic height was also obtained from the mesoscale model.

At each grid point, a one-dimensional RT calculation is performed. The resulting BTs are compared to the AMSU-B observed BTs. The nearest overpass over this area was at 1332 UTC. Note that there is a time difference of 30 minutes between the satellite overpass and the mesoscale model forecast. The satellite zenith angle corresponding to each AMSU-B pixel is obtained along with the data, and is used for the simulation of radiance at that grid point. The calculations are done only for one representative frequency per channel. A clear sky simulation was also done using the same inputs. In order to calculate the cloud signal, the clear sky results are subtracted from the simulation including clouds.

Note, that sub-pixel inhomogeneities (e.g., fractional cloud cover) are neglected here. They may account for a considerable part of the observed scatter between the RT simulations and the AMSU observations.

\section{Results of the comparison}

The figures showing the results are presented in an integrated form for all channels of AMSU-B. Fig. 3 shows the comparison results for all channels and Fig. 4 shows the signal due to ice and liquid clouds. The left column of Fig. 3 shows the observation, the middle column shows the simulation and the right column is a scatter plot between the observation and the simulation. The rows represent the channels in the order 16, 17, 18, 19, and 20. In Fig. 4, the top left and right columns shows the IWP and LWP, respectively. The remaining 

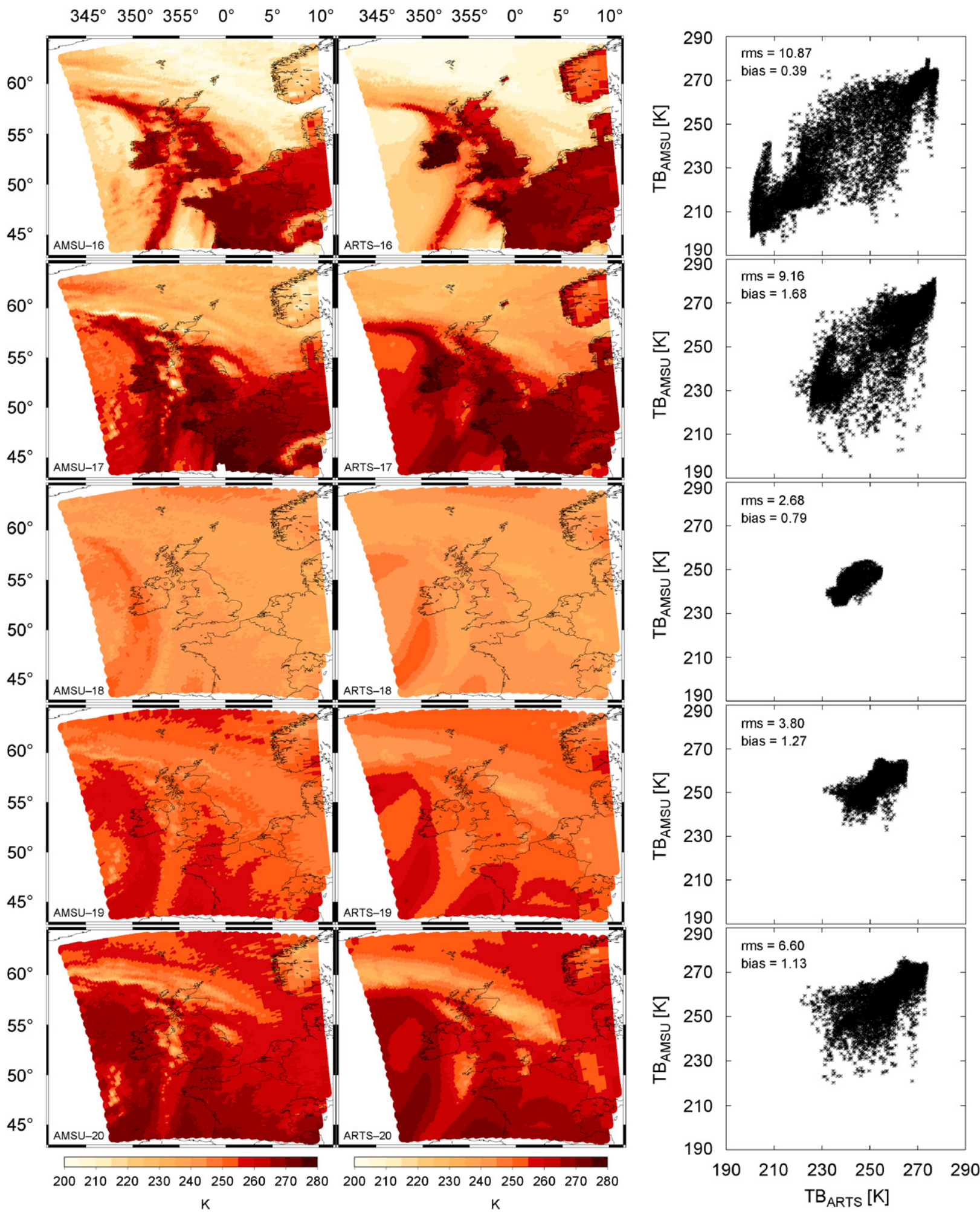

Fig. 3. Comparison results for all channels. The left panel shows the AMSU-B observation, the middle panel shows the ARTS simulation and the right panel shows the scatter plot between observation and simulation. The rows represent channels 16-20. 

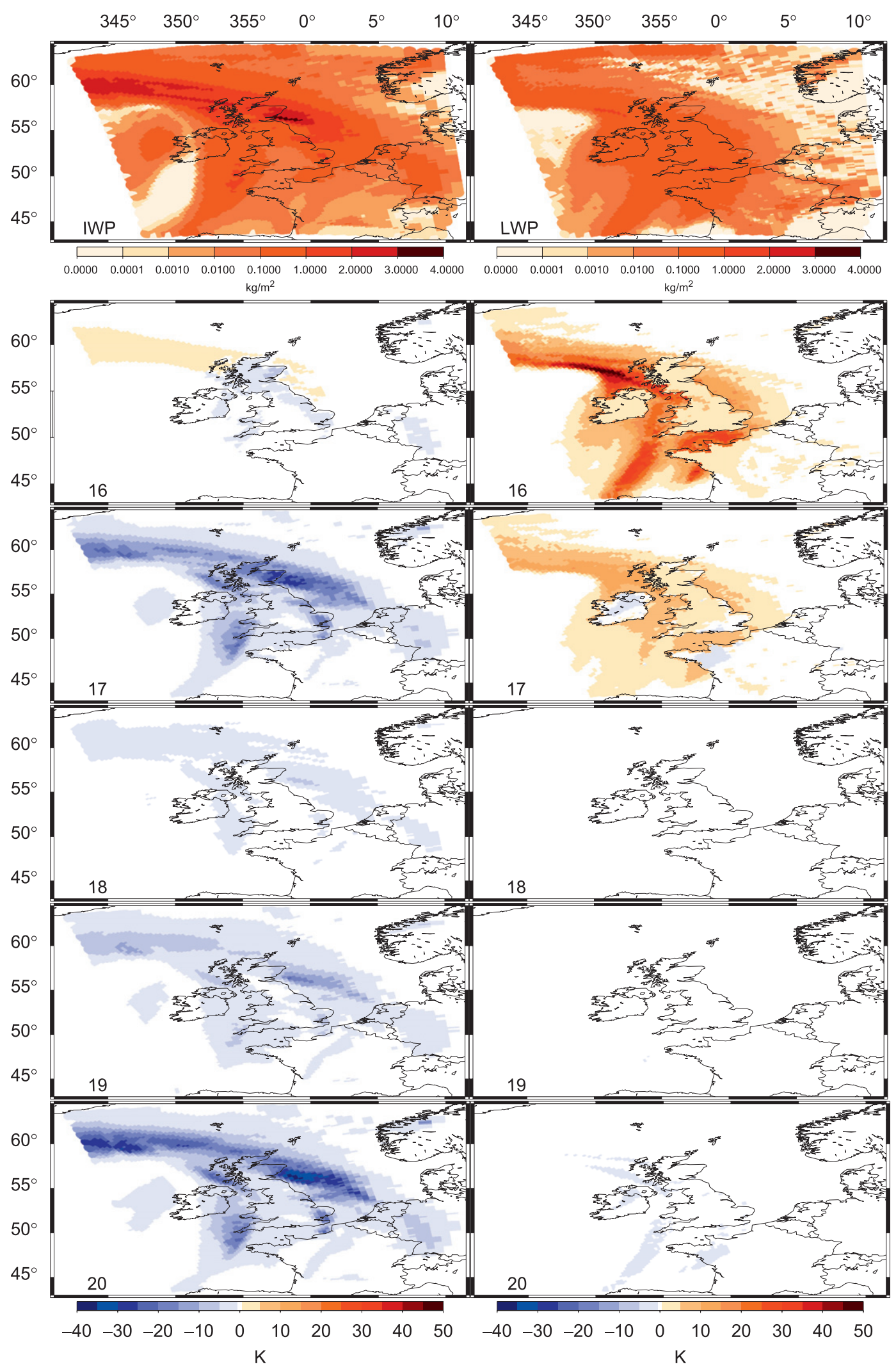

Fig. 4. The left and right panels on the first row shows the IWP and LWP, respectively. The remaining rows show the ice and liquid signal for AMSU channels 16-20. The ice cloud signal is in the left panel and the liquid cloud signal in the right panel. 
rows represent the ice cloud signal in the left column and liquid cloud signal in the right column for all the channels.

\subsection{Channels 16 and 17}

The left panel of the first two rows of Fig. 3 shows the BTs measured by AMSU channels 16 and 17, respectively. Both these channels can see deep into the atmosphere and are influenced by the surface. The high BT over land and the rather cold BT over the oceans, the Scandinavian region and the Alps can be seen clearly in the AMSU-B observation. This is because land is radiometrically warm compared to the ocean and the snow covered regions. The area of warm BTs over the ocean extending from the northwest of Spain up to the northwest of Ireland corresponds to the cloud region seen in the IR image (Fig. 2). This can be seen from the IWP and LWP distribution shown in Fig. 4 obtained from the mesoscale model. It is also illustrated by the cloud signal, especially that due to liquid clouds in Fig. 4 (second and third row for channels 16 and 17, respectively). Over these regions, the maximum BT difference between the clear sky simulation and the simulation including liquid clouds is about $50 \mathrm{~K}$ for channel 16 and $15 \mathrm{~K}$ for channel 17 . It can be seen from the left and middle panels of Fig. 3 that the area of warm BT seen in the AMSU-B observation is correctly reproduced in the simulation (first row for channel 16 and second row for channel 17). The maximum BT increase due to cloud is seen over the north of Ireland, which is also the region having the largest LWP. Over land, this increase is much smaller than over the ocean, as is expected.

For channel 16, from the ice cloud signal in Fig. 4 (second row first column) it can be seen that the presence of ice clouds does not change the BT of this channel as much as the liquid clouds. The maximum positive ice cloud signal is about $2 \mathrm{~K}$ and negative ice cloud signal is about $7 \mathrm{~K}$. The increase in BT over ocean regions is due to the low surface emissivity values. Low clouds emitting at a higher temperature against the radiatively cold surface background increase the contribution to the emission term. This increases the BT relative to the clear sky case. A BT depression is seen over land regions where the surface is radiometrically warmer compared to the ocean regions. Channel 17 , on the other hand is significantly affected by ice clouds. Apart from some spatial shifts coming from the mesoscale model inputs, ARTS produces similar BT depressions as seen in the observation. A maximum depression of about $30 \mathrm{~K}$ is observed at regions of very high IWP.

As expected there are some discrepancies between the observation and the simulation. For example, over the Alps and over Norway, the simulation is colder than the observation due to the assumption of surface emissivity values. The scatter plot of AMSU observed BT against ARTS simulated BT shows that at several points ARTS simulates higher BTs compared to the AMSU-B observations. Besides the differences arising from the simplistic treatment of land surface emissivity there are several reasons for differences on a pixel by pixel comparison. Firstly, the position and amplitude of the NWP fields are obviously not precisely the same as the real observed fields. These differences we wish to exploit in order to validate NWP models. Furthermore the observations are sensitive to scales the NWP model cannot represent due to the lower resolution. In the RT simulations assumptions have been made about the cloud microphysics which may not be equally applicable to every field of view. However the distribution of observed and calculated BTs is very similar. It appears likely that positional and amplitude errors are the most significant source of difference, which gives hope that we may be able to exploit the cloud and rain information in AMSU-B observations in NWP data assimilation systems in the future.

\subsection{Channels 18,19 , and 20}

For all three channels 18, 19, and 20, Fig. 3 shows that the simulations in the presence of clouds match well with the observations over the whole region. By comparing the observation to the simulation and to the cloud signal it can be seen that ARTS in combination with the NWP model fields correctly simulates the cloud effects. For example, for channel 20, from Fig. 3 (sixth row first column), the very cold BTs over the north Atlantic in the observation are correctly represented in the simulation. The temporal difference of $30 \mathrm{~min}$ between the observation and model forecast has given rise to a spatial shift in the ice cloud features, like the cold BTs over northern parts of Germany which are not present in the observation. The magnitude of the BT depressions seen in the simulation is closely associated with the values of the IWP from the mesoscale model. 
For all these channels, over some regions, e.g., off the east coast of Northern England, it can be seen from Fig. 4 that the simulations overestimate the BT depression. This is either because the mesoscale model overestimates the ice concentration over that region or because the assumptions on the particle size distribution and shape does not correspond to the real scenario.

Among channels 18, 19, and 20, channel 18 has the minimum influence from clouds, and channel 20 has the maximum influence from clouds. From the ice cloud signal in Fig. 4, it can be seen that for channel 18, the maximum depression is about $8 \mathrm{~K}$. Channel 19 , which is sensitive to comparatively lower altitudes, has a maximum depression of about $20 \mathrm{~K}$, and channel 20 , which is the channel most sensitive to clouds, has a BT depression of about $40 \mathrm{~K}$.

For these three channels, liquid clouds decrease the BT with respect to the clear sky case. Channel 18 is not at all sensitive to liquid clouds because the liquid water clouds are located below its sounding altitude. The maximum depression simulated due only to liquid clouds is only about $0.01 \mathrm{~K}$. For channels 19 and 20 also, the impact of liquid clouds on the BT is very small, the maximum depression is about $1 \mathrm{~K}$ for channel 19 and about $5 \mathrm{~K}$ for channel 20 . For channel 20, over dry regions, liquid water can lead to a slight increase in BT (about $0.1 \mathrm{~K}$ ) compared to the clear sky case. This effect can be seen over radiatively cold surfaces such as ocean.

Overall, the agreement between simulated and observed radiances is remarkably good, considering the rather coarse assumptions that were made in the model setup. For example, the agreement is comparable, if not slightly better, to the agreement found by Meirold-Mautner et al. [16] who did a similar comparison study with more detailed model fields (using six different water species with different microphysical assumptions). To make a direct comparison with the results of Meirold-Mautner et al. [16, Figure 5] we show the histogram of the difference between modelled and observed BTs for channel 18 in Fig. 5.

Furthermore, the agreement between the simulation shown here and the observations is considerably better than the agreement between RTTOV simulations and observations for the same scene that are presented and discussed by Doherty et al. [29].

This most likely is caused by the microphysical assumptions, and not by differences in the RT algorithms themselves, since studies have shown that different scattering RT algorithms arrive at rather similar solutions if they are set up identically (e.g. [30,31]).

\subsection{Impact of ice particle size distribution, shape, and orientation on radiances and on polarisation}

While analysing the results, it has to be kept in mind that one of the big uncertainties in the simulations presented above is associated with the assumptions on cirrus microphysical properties. This includes the

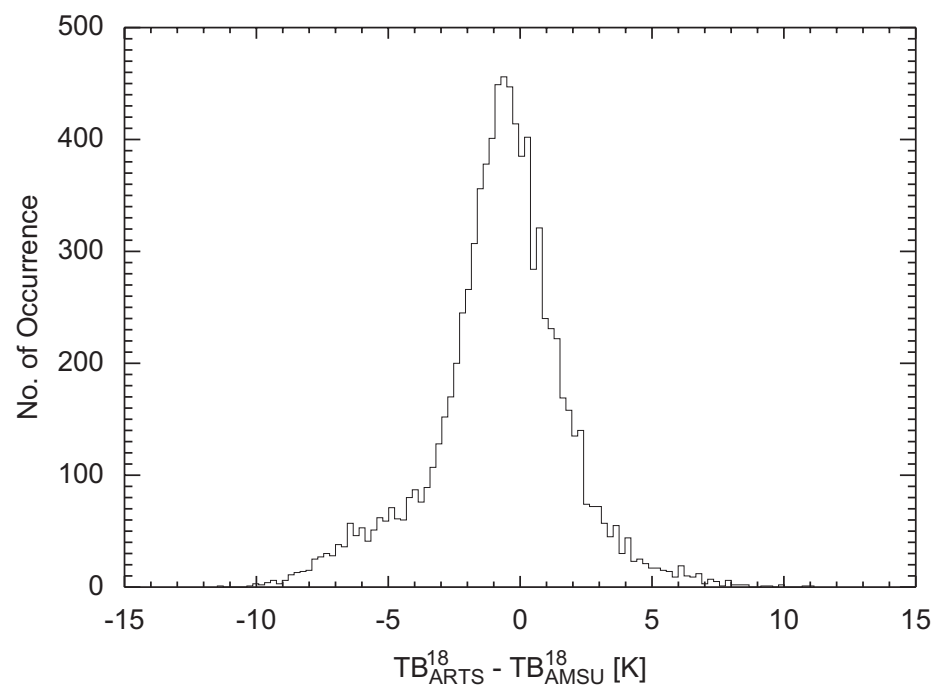

Fig. 5. Histogram of difference in modelled and observed brightness temperatures for channel 18. 
uncertainties in the size distribution, shape and orientation of ice crystals. For oriented particles, the contribution of polarisation also has to be taken into account. It is well known that ice particles are not spherical and that they are not randomly oriented [32-34]. Here we try to give an estimate of the error that can be introduced by the assumptions related to particle size distribution, shape and orientation.

In all the simulations, the size distribution of ice particles was according to the parameterisation by McFarquhar and Heymsfield [27], developed mainly for tropical cirrus. In the microwave range, increasing the size of the particle while keeping the ice water content constant, leads to a higher BT depression [9]. This is because, the extinction and scattering coefficients increase with particle effective size. In order to understand the impact of the size distribution on AMSU-B radiances, two simulations were done. In the first simulation for all profiles, ice particles at all atmospheric layers follow a gamma distribution with effective radius (which is a measure for the mean size in a particle population [35] of $50 \mu \mathrm{m}$ and all the particles were spherical. The maximum BT depression for channel 20 was $9 \mathrm{~K}$. Increasing the particle effective radius to $100 \mu \mathrm{m}$, while keeping the same ice water content, the maximum BT depression was $33 \mathrm{~K}$. These maximum depressions were found at the region of maximum IWP, about $3 \mathrm{~kg} \mathrm{~m}^{-2}$. The difference between the BTs in the two simulations is plotted as a function of IWP in Fig. 6. The mean increase in BT was $3.5 \mathrm{~K}$ and the root mean square (RMS) difference was $5.5 \mathrm{~K}$. This is because the number of profiles that have low IWP, where the size effect is less than a few $\mathrm{K}$, is much larger than the number of profiles that have high IWP.

Evans and Stephens [36] found that particle shape has a significant impact on simulated sub-mm wave radiances. It is not clear, whether this applies also to the AMSU measurements, which are lower in frequency. Thus, to estimate the impact of the asphericity of ice particles, a simulation was done assuming randomly oriented ice particles of aspect ratio four (oblate spheroid); all other parameters remain exactly as explained in Section 5. The choice of random orientation and modest aspect ratio is supported by microwave limb sounding data [37], which indicate that effective orientedness and aspect ratios are modest, even though individual ice crystals may be strongly aspherical and have a strong orientation preference.

Channel 20 showed the largest sensitivity to the degree of asphericity of the ice particles. It was found that for this channel, the mean BT difference between this simulation and the simulation assuming spherical particles is about $0.26 \mathrm{~K}$ (with maximum of about $1.8 \mathrm{~K}$ ) and the RMS value is about $0.4 \mathrm{~K}$.

In order to determine the impact of oriented ice particles on AMSU-B radiances, a simulation where the non-spherical particles were horizontally aligned but azimuthally randomly oriented, was also performed.

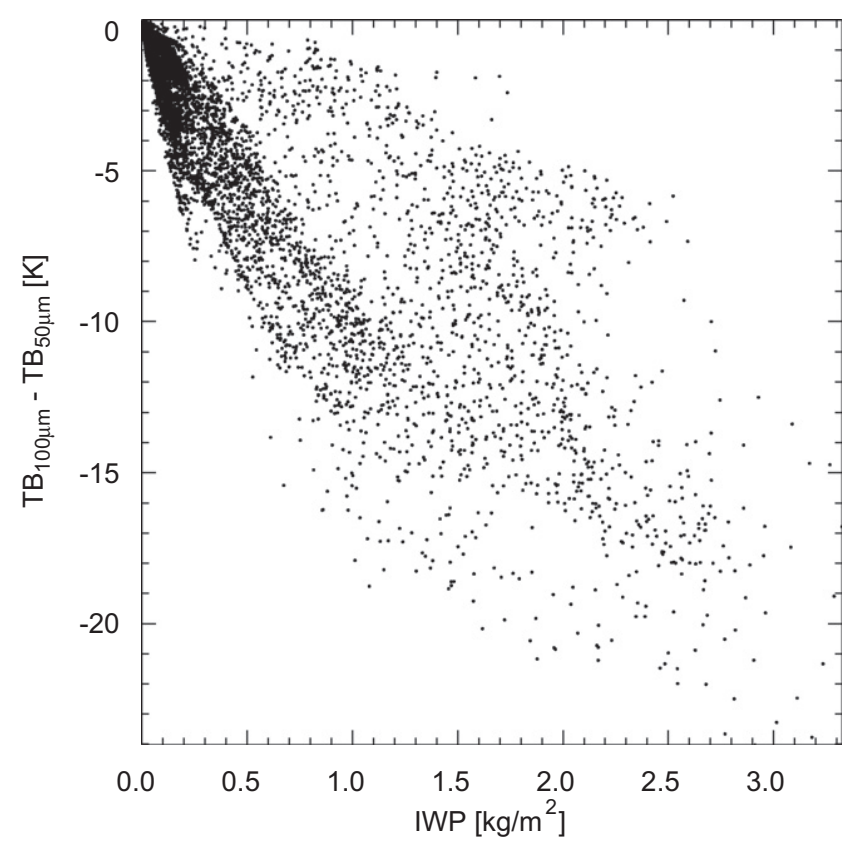

Fig. 6. The difference in BT between simulations where the effective radii are 50 and $100 \mu \mathrm{m}$ as a function of IWP. 
This simulation was also done to estimate the polarisation signal due to the oriented ice particles. In interpreting the results, it should be kept in mind that this is probably a rather extreme case of asphericity. Although individual cloud ice particles have high aspect ratios, there is a lot of orientation averaging for the bulk optical properties, even if particles have a preferred horizontal orientation. Davis et al. [37] found that an effective aspect ratio of 1.2 for horizontally aligned oblate spheroids is consistent with the polarisation signal in microwave limb sounder (MLS) observations.

The difference in BT between the previous simulation of randomly oriented non-spherical particles and the simulation with oriented particles was calculated. For channel 20, the mean difference was about $3 \mathrm{~K}$ and the RMS was about $5.2 \mathrm{~K}$. Here also, like in the case of size distribution, at very high IWPs, the difference between the simulations is very high. For the profile corresponding to the highest IWP, the difference was about $18 \mathrm{~K}$. The results indicate the extent of error that can be introduced by the assumption of randomly oriented spherical particles. Maximum differences are associated with regions of very high IWP.

The impact of the particle shape depends strongly on the assumed aspect ratio. In our simulations, the impact of the shape was modest, because the assumed aspect ratio was modest. This is consistent with observations, e.g. [37]. While individual ice particles have high aspect ratios, these data indicate that average aspect ratios, which are the relevant ones for RT, are modest.

It is not possible to compare the polarisation signal of the RT model with the observation since AMSU-B does not measure polarisation. As mentioned in Section 4, it measures a mixed polarisation. It is shown in Emde et al. [17] that the linear polarisation component $Q$ of the Stokes vector is not very significant for downlooking angles and that it increases for non-spherical particles compared to spherical particles. Also, $Q$ is higher for horizontally aligned particles than for randomly oriented particles. Therefore, here the result from the previous simulation assuming cylinders having an aspect ratio of four which are horizontally aligned and azimuthally randomly oriented, are used to calculate the linear polarisation, $Q$. The particles follow the McFarquhar and Heymsfield [27] size distribution.

The maximum value for $Q$ obtained is about $8 \mathrm{~K}$. The sign of $Q$ is always positive and is consistent with the results presented in Emde et al. [17] for horizontally aligned particles observed at down-looking angles. At nadir, $Q$ is zero and increases towards the maximum off-nadir viewing angle, $50^{\circ}$. The maximum values of $Q$ depend also on IWP. If the line of sight is highly off-nadir, a small increase in IWP can lead to a large increase in $Q$. This is demonstrated in the left panel of Fig. 7. The information on the zenith angle associated with each pixel is used to sort $Q$ accordingly. For example, the points corresponding to nadir were chosen considering only those pixels whose viewing angles are between nadir and $1^{\circ}$ off-nadir.
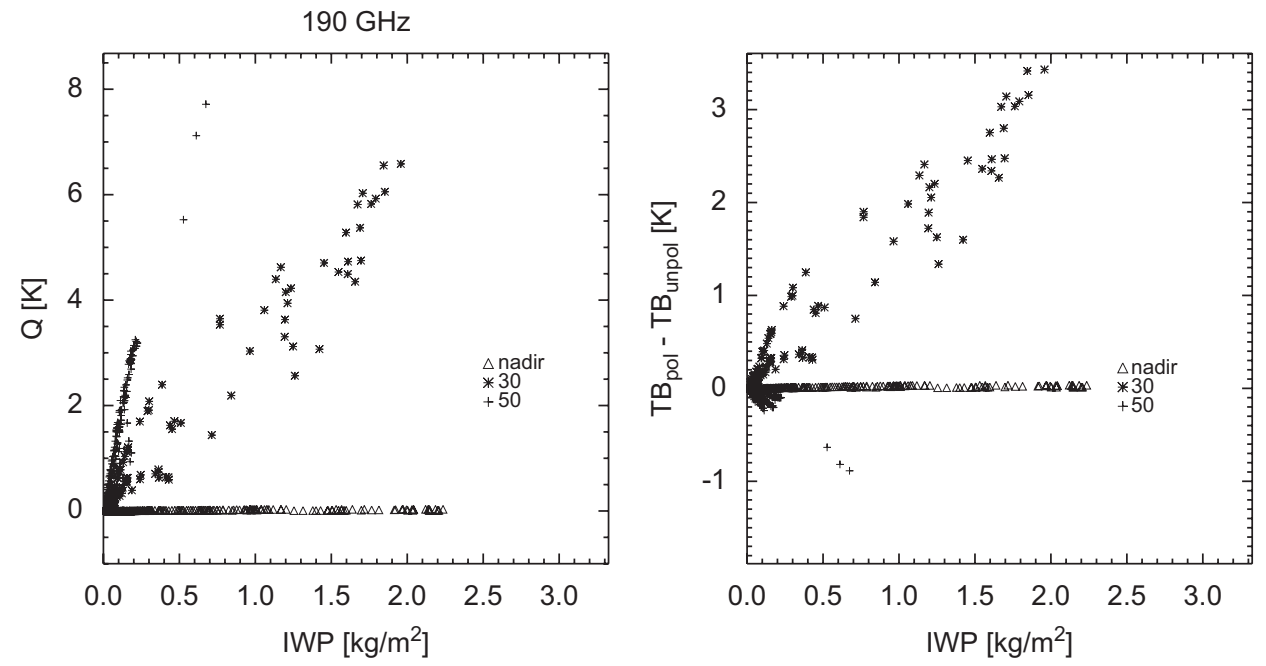

Fig. 7. The left plot shows the polarisation difference $Q$ as a function of IWP for $190 \mathrm{GHz}$. The right plot shows the difference $I_{\text {mix }}-I$ as a function of IWP. The symbols represent the viewing angles indicated in the legend. 
The contribution of $Q$ to the total intensity measured by AMSU-B can be calculated using Eq. (1), if the total intensity $I$ and the polarisation difference $Q$ are known:

$$
I_{\text {mix }}=(I+Q) \sin ^{2} \theta_{\mathrm{p}}+(I-Q) \cos ^{2} \theta_{\mathrm{p}} .
$$

Fig. 7 shows the difference, $I_{\operatorname{mix}}-I$, where $I$ is the unpolarised radiation. When $Q$ is zero, $I_{\operatorname{mix}}=I$. At angles very close to nadir, $Q$ is zero. Therefore the differences are zero, as indicated by the ' $\triangle$ ' symbols in the plot. The difference $I_{\mathrm{mix}}-I$ can be conveniently written as

$$
I_{\text {mix }}-I=-Q \cos ^{2} \theta_{\mathrm{p}} \text {. }
$$

As the viewing angle becomes more off-nadir, $Q$ increases. If AMSU-B were a limb-viewing instrument, then exactly at limb, $\theta_{\mathrm{p}}$ is equal to zero and the difference $I_{\mathrm{mix}}-I$ is $-Q$. As the viewing angle moves away from nadir $\left(\theta_{\mathrm{p}}=90^{\circ}\right)$, the difference $I_{\text {mix }}-I$ increases up to a viewing angle of $45^{\circ}$ off-nadir. The difference $I_{\text {mix }}-I$ is positive as well. This is shown by the ' $*$ ' symbols in Fig. 7. Exactly, at $45^{\circ}$ off-nadir viewing angle, the difference should be again zero, because here $\theta_{\mathrm{p}}$ in Eq. (3) is $45^{\circ}$. Beyond $45^{\circ}$, the difference becomes negative as is seen by the ' + ' symbols. This is because $Q$ increases and the second term in Eq. (2) starts dominating. At viewing angles beyond $45^{\circ}$, the polarisation increases the BT, i.e., decreases the BT depression. The maximum difference is about $3 \mathrm{~K}$.

\section{Summary, conclusion, and outlook}

The aim of this study is to estimate the effect of clouds on AMSU-B observations. Observations of all AMSU-B channels are simulated for a strong frontal system over the UK using the RT model ARTS. The input profiles of pressure, temperature, humidity, and ice and liquid water content required for the RT simulation are taken from the UK Met Office mesoscale model forecast. The simulated BTs are compared to the AMSU observations. The results of the comparison show that for all channels, as expected, cloud effects cannot be neglected. The differences between simulation and observation are mainly due to the model input, e.g. they show the differences between the model forecast and the real cloud conditions. Such simulations may be used in future studies for validation of NWP models.

Among all the channels, channel 16 is most affected by the presence of liquid clouds. A maximum BT increase of about $50 \mathrm{~K}$ compared to the clear sky case was found over regions of high LWP. Ice clouds have only a very small effect on the BT of this channel. Over ocean regions, the presence of ice clouds increases the BT compared to the clear sky case because the absorption against a cold surface background makes the emission term more dominant, thus increasing the BT. Over land, where the surface is radiometrically warmer, the extinction term becomes dominant, thus leading to a BT depression.

Among the sounding channels, channel 20 is the one most affected by ice clouds. The maximum difference to the clear sky simulation is close to $40 \mathrm{~K}$ in a region of very high IWP. There is a small enhancement due to liquid clouds over very cold regions. This channel is sensitive to lower layers of the atmosphere (compared to channels 18 and 19) which is why the impact is the highest here. There are some discrepancies between the observation and simulation. Over some regions the simulation overestimates the BT depression.

A possible reason for the discrepancy is attributed to the assumptions related to cloud microphysical properties. Since the mesoscale model does not give information on cloud microphysical properties, there are many free input parameters for the RT simulation. These include the size distribution, the shape and the orientation of ice and liquid particles. The impact of the size distribution was found to be the most significant followed by shape and orientation. For randomly oriented particles, the effect of shape was found to be negligible for the frequencies and particle types considered.

The impact of polarisation was also investigated, which shows a maximum difference of about $3 \mathrm{~K}$ compared to the unpolarised BTs. To put this value into perspective, one should take into account that the considered case was most likely a rather extreme case of asphericity, which means that average polarisation effects are likely to be much smaller than this value.

The study shows is that it is still unclear which microphysical assumptions on cloud ice particles are most appropriate to represent them in microwave RT simulations. The comparison to other case studies shows that more elaborate microphysical assumptions do not necessarily lead to better agreement with observations. 
Sensitivity studies about which parameters are most crucial suffer from a lack of comparability, since they use completely different model frameworks. For example, in Meirold-Mautner et al. [16] it was found that significant discrepancies were related to the snow parameterisation in the RT code as interpreted from the model fields. The mesoscale model used in this study uses a scheme in which all frozen water including snow, pristine ice crystals and rimed particles are considered as one hydrometeor type, namely ice. Larger ice particles in the McFarquhar and Heymsfield [27] ice particle size distribution can give a similar scattering signal as snow. This poses a challenge to the cloud RT modelling community: on one hand, we need more detailed and quantitative studies to really be able to predict the microwave cloud signal accurately, and to do good cloud retrievals from measured radiances. On the other hand, results of detailed studies currently suffer from a lack of generality and transferability to other model setups.

\section{Acknowledgements}

Many thanks to S.J. English, Met Office, UK for fruitful discussions. We thank Oliver Lemke for programming assistance and technical support. Furthermore, thanks to the ARTS Radiative Transfer community, many of whom have indirectly contributed by implementing features to the ARTS model, in particular, thanks to Patrick Eriksson, at the Chalmers University. We also thank Michael Mishchenko for the T-matrix program, Stephan Warren for the refractive index program and Cory Davis for the PyARTS software. Thanks also to Brett Candy who helped obtain the UKMES data for the case study. This study was funded by the German Federal Ministry of Education and Research (BMBF), within DLR project SMILES, Grant 50 EE 9815, and within AFO2000 project UTH-MOS, Grant 07ATC04. It was co-funded by ESA project UTLS, ESTEC Contract No. $15457 / 02 / \mathrm{NL} / \mathrm{MW}$. It is a contribution to COST Action 723 'Data Exploitation and Modeling for the Upper Troposphere and Lower Stratosphere'. The authors are also grateful to the NWP-SAF.

\section{References}

[1] Rosenkranz PW. Retrieval of temperature and moisture profiles from AMSU-A and AMSU-B measurements. IEEE Geosci Remote Sensing 2001;39(11):2429-35.

[2] Sohn B-J, Chung E-S, Schmetz J, Smith EA. Estimating upper-tropospheric water vapor from SSM/T-2 satellite measurements. J Appl Meteorol 2003;42:488-504.

[3] English SJ, Renshaw RJ, Dibben PC, Smith AJ, Rayer PJ, Poulsen C, et al. A comparison of the impact of TOVS and ATOVS satellite sounding data on accuracy of numerical weather forecasts. Q J R Meteorol Soc 2000;126:2911-31.

[4] Greenwald TJ, Christopher SA. Effect of cold clouds on satellite measurements near 183 GHz. J Geophys Res 2002;107(D13).

[5] Mueller BM, Fuelberg HE, Xiang X. Simulations for the effects of water vapor, cloud liquid water and ice on AMSU moister channel brightness temperatures. J Appl Meteorol 1994;33:1133-54.

[6] Weinman J. The effects of cirrus clouds on $118 \mathrm{GHz}$ brightness temperatures. J Geophys Res 1998;93(D9):11059-62.

[7] Burns BA, Wu X, Diak GR. Effects of precipitation and cloud ice on brightness temperatures in AMSU moisture channels. I3ETGRS 1997;35(6):1429-37.

[8] Hong G, Heygster G, Miao J, Kunzi K. Detection of tropical deep convective clouds from AMSU-B water vapor channels measurements. J Geophys Res 2005;110(D9).

[9] Sreerekha TR. Impact of clouds on microwave remote sensing. PhD thesis, University of Bremen; 2005.

[10] Kerridge B, Jay V, Reburn J, Siddans R, Latter B, Lama F, et al. Consideration of mission studying chemistry of the UTLS, final report. Technical Report, ESTEC Contract No. 15457/01/NL/MM; 2004.

[11] Emde C. A polarized discrete ordinate scattering model for radiative transfer simulations in spherical atmospheres with thermal source. PhD thesis, University of Bremen; 2005, ISBN 3-8325-0885-4.

[12] Hoepfner M, Emde C. Comparison of single and multiple scattering approaches for the simulation of limb-emission observations in the mid-IR. JQSRT 2005;91(3):275-85.

[13] Mendrok J. The sartre model for radiative transfer in spherical atmospheres and its application to the derivation of cirrus cloud properties. PhD thesis, FU Berlin; 2006.

[14] Wu X, Diak GR, Hayden CM, Young JA. Short-range precipitation forecasts using assimilation of simulated satellite water vapor column cloud liquid water amounts. Mon Weather Rev 1995;123:347-65.

[15] Tripoli GJ. A nonhydrostatic mesoscale model designed to simulate scale interaction. Mon Weather Rev 1992;120:1342-59.

[16] Meirold-Mautner I, Prigent C, Defer E, Pardo JR, Chaboureau J-P, Pinty J-P, et al. Radiative transfer simulations using mesoscale cloud model outputs: comparisons with passive microwave and infrared satellite observations for mid-latitudes. J Atmos Sci 2007;64:1550-68. 
[17] Emde C, Buehler SA, Davis C, Eriksson P, Sreerekha TR, Teichmann C. A polarized discrete ordinate scattering model for simulations of limb and nadir longwave measurements in 1D/3D spherical atmospheres. J Geophys Res 2004;109(D24).

[18] Davis C, Emde C, Harwood R. A 3D polarized reversed Monte Carlo radiative transfer model for mm and sub-mm passive remote sensing in cloudy atmospheres. IEEE Trans Geosci Remote 2005;43(6):1096-101.

[19] Mishchenko MI, Travis LD. Capabilities and limitations of a current FORTRAN implementation of the T-matrix method for randomly oriented rotationally symmetric scatterers. JQSRT 1998;60:309-24.

[20] Warren SG. Optical constants of ice from the ultraviolet to the microwave. Appl Opt 2000;23:1206-25.

[21] Lorenc AC, Ballard SP, Bell RS, Ingleby NB, Andrews PLF, Baker DM, et al. The Met Office global three dimensional variational data assimilation scheme. Q J R Meteorol Soc 2000;126(570):2991-3012.

[22] Saunders RW, Hewison TJ, Stringer SJ, Atkinson NC. The radiometric characterization of AMSU-B. IEEE Trans Microwave Theory Tech 1995;43(4):760-71.

[23] John VO, Buehler SA. The impact of ozone lines on AMSU-B radiances. Geophys Res Lett 2004;31:L21108.

[24] Anderson GP, Clough SA, Kneizys FX, Chetwynd JH, Shettle EP. AFGL atmospheric constituent profiles (0-120 km). Technical Report TR-86-0110, AFGL; 1986.

[25] Rosenkranz PW. Water vapor microwave continuum absorption: a comparison of measurements and models. Radio Sci 1998;33(4):919-28 [correction in 34, 1025, 1999] 〈ftp://mesa.mit.edu/phil/lblrt $\rangle$.

[26] Rosenkranz PW. Absorption of microwaves by atmospheric gases. In: Janssen MA, editor. Atmospheric remote sensing by microwave radiometry. New York: Wiley; 1993. p. 37-90 〈ftp://mesa.mit.edu/phil/lblrt $\rangle$.

[27] McFarquhar GM, Heymsfield AJ. Parameterization of tropical cirrus ice crystal size distribution and implications for radiative transfer: results from CEPEX. J Atmos Sci 1997;54:2187-200.

[28] English SJ, Hewison T. A fast generic millimetre wave emissivity model. In: Proceedings of the SPIE conference, vol. 3503 ; 1998.

[29] Doherty AM, Sreerekha TR, O'Keeffe UM, English SJ. Ice hydrometeor microphysical assumptions in radiative transfer models at AMSU-B frequencies. Q J R Meteorol Soc 2007;133(626):1205-12.

[30] Battaglia A, Davis CP, Emde C, Simmer C. Microwave radiative transfer intercomparison study for 3-D dichroic media. JQSRT 2007;105(1):55-67.

[31] Deeter MN, Evans KF. A hybrid Eddington-single scattering radiative transfer model for computing radiances from thermally emitting atmospheres. JQSRT 1998;60(4):635-48.

[32] Chepfer H, Brogniez G, Goloub P, Breon FM, Flamant PH. Observations of horizontally oriented ice crystals in cirrus clouds with POLDER-1/ADEOS-1. JQSRT 1999;63:521-43.

[33] Chepfer H, Goloub P, Riedi J, De Haan JFH, Hovenier JW, Flamant PH. Ice crystal shapes in cirrus clouds derived from POLDER/ ADEOS-1. J Geophys Res 2001;106(D8):7955-66.

[34] Breon F-M, Dubrulle B. Horizontally oriented plates in clouds. J Atmos Sci 2004;61:2888-98.

[35] Wyser K. The effective radius in ice clouds. J Climate 1998;11:1793-801.

[36] Evans KF, Stephens GL. Microwave radiative transfer through clouds composed of realistically shaped ice crystals. Part II. Remote sensing of ice clouds. J Atmos Sci 1995;52:2058-72.

[37] Davis CP, Wu DL, Emde C, Jiang JH, Cofield RE, Harwood RS. Cirrus induced polarization in 122 GHz Aura Microwave Limb Sounder radiances. Geophys Res Lett 2005:32. 